\title{
Moving downstream
}

DOI:

10.1038/nrc2002

\section{URLs \\ Links \\ VEGF \\ http://www.ncbi.nlm.nih.gov/ entrez/query.fcgi?db=gene\&c $\mathrm{md}=$ Retrieve\&dopt=full report\&list_uids $=7422$}

AKT1

http://www.ncbi.nlm.nih.gov/ entrez/query.fcgi?db=gene\&c $\mathrm{md}=$ Retrieve\&dopt=full report\&list_uids $=207$

\section{mTOR}

http://www.ncbi.nlm.nih.gov/ entrez/query.fcgi?db=gene\&c $\mathrm{md}=$ Retrieve\&dopt=full_ reportElist_uids=2475
Vascular endothelial growth factor (VEGF) increases tumour angiogenesis, but now Laura Benjamin and colleagues have looked downstream of VEGF and shown that activation of the Akt pathway - one of the pathways activated in response to VEGF - can recapitulate the effects of VEGF on tumour blood vessels.

To investigate the role of endothelial-cell-autonomous Akt activation in blood vessels in an otherwise normal microenvironment, the authors used transgenic mice that expressed a tetracycline-repressible myristoylated AKT1 (myrAKT1) that is constitutively active specifically in endothelial cells. They observed distinct differences in the vasculature of mice with endothelial expression of myrAKT1 compared with those without myrAKT1 induction. The surface area of the skin of mice with activated myrAKT1 was covered by twice as many blood vessels, and these vessels had larger diameters and were more tortuous. Furthermore, the mice had increased blood volume, and there was a trend towards increased permeability of the myrAKT1 vessels. These abnormal blood vessels that mimic tumour vasculature but are formed in the absence of a tumour are called 'pathological blood vessels'.

Is the continued activation of AKT1 required to maintain these pathological blood vessels? The authors activated myrAKT1 expression in mice by withdrawing tetracycline and then restored tetracycline to suppress myrAKT1 expression. They found that the effects of myrAKT1 on blood vessels were completely reversed when myrAKT1 expression was suppressed, indicating that the vessels were dependent on sustained AKT1 signalling.

One downstream effector of Akt is mammalian target of rapamycin (mTOR), and the mTOR inhibitor rapamycin is currently being investigated as an anticancer agent. Treating mice with rapamycin at the same time as tetracycline was withdrawn to induce myrAKT1 expression blocked the formation of pathological blood vessels. The inhibition of mTOR by rapamycin not only blocks signalling downstream of mTOR, but also reduces Akt activation. The authors attribute the observed effects in endothelial cells to this inhibition of the Akt pathway. They also injected tumour cells that expressed VEGF into nude mice, and showed that rapamycin treatment significantly reduced tumour growth, endothelial Akt activation and the permeability of tumour blood vessels.

The work by Benjamin and colleagues highlights the importance of the Akt-mTOR pathway in angiogenesis. Furthermore, it indicates that the inhibition of this pathway, for example by rapamycin, is a valid therapeutic strategy for tumours with increased angiogenesis. The ability of rapamycin to inhibit pathological angiogenesis and vascular leak might also facilitate the more effective delivery of chemotherapeutic agents to tumours. As VEGF-inhibitors are already being used in patients with various cancers, it will be interesting to see whether the inhibition of the Akt-mTOR pathway replicates or amplifies the effects of VEGF inhibition.

Sarah Seton-Rogers

ORIGINAL RESEARCH PAPER Phung, T. L. et al. Pathological angiogenesis is induced by sustained Akt signalling and inhibited by rapamycin. Cancer Cell 10, 159-170 (2006)

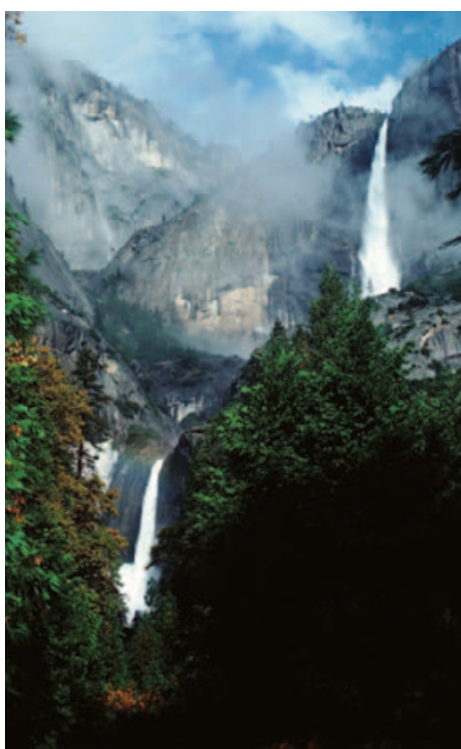

